Volume 8, No.6, November - December 2019

International Journal of Advanced Trends in Computer Science and Engineering

Available Online at http://www.warse.org/IJATCSE/static/pdf/file/ijatcse29862019.pdf

https://doi.org/10.30534/ijatcse/2019/29862019

\title{
Multi-Objective Optimization for Worldview Image Segmentation Funded on the Entropies of Tsallis and Rényi
}

\author{
Salah Eddine MECHKOURI ${ }^{1}$, Saleh EL JOUMANI ${ }^{2}$, Rachid ZENNOUHI ${ }^{3}$, Lhoussaine MASMOUDI $^{4}$ \\ ${ }_{1,2,3} \& 4$ University Mohammed V in Rabat, Faculty of Science, Department of Physics. Morocco \\ ${ }^{1}$ salahmechkouri@gmail.com; \\ ${ }^{2}$ jomanisalh@gmail.com; \\ ${ }^{3}$ r.zennouhi@gmail.com; \\ ${ }^{4}$ lhmasmoudi@fsr.ac.ma
}

\begin{abstract}
The article describes a new method of threshold satellite image, based on the optimization multi-objective for segmentation of Worldview images and funded on the Tsallis and the Rényi entropies, allowing the evolution of the satellite image classification. Owing to the goal of achieving a large classifying all unclassified pixels by the previous method in 2017 in our research laboratory. An improved analysis and a multi-objective optimized thresholding method was proposed. Firstly, we are calculate the optimal thresholds with respect to the criteria retained such as the Tsallis criterion and the Rényi criterion. Lastly, we are challenging the performance of our method to that developed previously in 2017. The new method effectiveness evaluation confirmed by the calculation of the evaluation criterion related on both the Levine and Nazif criterion and the Mean Squared Error criterion. The results obtained by our approach were very satisfactory. It was been shown that the method overcomes the difficulties of the method previously developed in 2017 and obtained results that are more precise. In particular, for synthetic images, the segmentation accuracy increases by $81.16 \%$ and for the satellite images, the segmentation also improves enormously, and the accuracy of the overall classification of Worldview images increases by $97.21 \%$. Therefore, the new method based on multi-objective optimization contribute significantly to performance.
\end{abstract}

Key words: Optimization multi-objective, Segmentation, Tsallis and Rényi entropies, Worldview Image.

\section{INTRODUCTION}

The extraction and the analysis of image data is an essential step for image segmentation. In the current article, we propose an improved thresholding technique founded on Tsallis and Rényi entropies for Worldview image segmentation.
First, we explore the centers of gravity of each gray-scale image region and the corresponding thresholds in their histograms according to Mechkouri et al. [1, 2], Sarala et al. [3] and Zennouhi et al. [4]. In order to reveal the opportunity of our proposed method, we compared it with that developed in 2017 by EL Joumani et al [5]. Secondly, we will evaluate our algorithm by the image segmentation of the desert environment.

The empirical results of our method show that the Tsallis and Rényi entropies offer competitive segmentation performances compared to the results of the formerly method [5]. Therefore, they depict the validity of our method; we will test and apply them to synthetic images and evaluate our Worldview image segmentation algorithm of the desert environment.

Our article designed as follow: The first part of this article offering the description of multi-objective optimization approach. The second one introduces the Levine and Nazif criterion, and the mean of squared error criterion -MSE-. The third one presents the Worldview image we used in this study. The quarter one exposes the experimental outcomes and the discussion. The last one gives the conclusion.

\section{DESCRIPTION OF THE METHOD}

Optimization theory derives from multi-objective optimization while allowing the concomitant optimization of several design objectives. The resolution of the multi-objective optimization problematic done in the same way as a classic problem with a lone objective. The objective is to categorize a set of values for the design variables that simultaneously optimize functions for multiple purposes.

Generally, in the case of simple objective optimization, the solution obtained by the optimization of each separate objective cannot represent a possible solution for the multi-objective problem. 


\subsection{Objective Function}

The objective function proposed by Nakib et al. [6] allows using the multi-objective optimization approach to find the optimal thresholds of some criteria. Since then, the optimal threshold for each criterion does not approve the conclusion of a satisfactory image segmentation. Nakib define a multi-objective function applied a multilevel image thresholding (such as medical images) as follows:

$$
\operatorname{MOBJ}(T)=w_{1} \operatorname{MVAR}(R(T))+w_{2} E(T)
$$

This function has evidently been successful, but it showed its limits. Hither, we propose optimal thresholds that allow optimizing a set of criteria. The method of thresholding built on two criteria:

- Tsallis entropy criterion and

- Rényi entropy criterion

Accordingly, to increase the quality of the segmentation we suggest to modify this objective function by replacing the criteria of the moderate variance in the class and the global probability of error by the criteria of the entropy of Tsallis and the entropy of Rényi successively, we can take into account as follows:

$$
N e w_{-} M O B J(T)=w_{1} s_{q}(T)+w_{2} H_{\alpha}(T)
$$

Where

- $S_{q}(T)$ the Tsallis's entropy is developed in the subsection $\$ 2.2$;

- $H_{\sigma}(T)$ the Rényi's entropy is developed in the subsection $\S 2.3$;

- $\mathrm{T}$ is the vector of thresholds: $0<\mathrm{T}_{1}<\mathrm{T}_{2}<\ldots<\mathrm{T}_{\mathrm{d}-1}<$ 255

- $w_{1}=1-w_{2}$;

- $w_{2}=\frac{\Sigma_{i=1}^{d} \sigma_{i}^{3}}{\sigma_{\text {Hiringram }}^{2}}$

- $\mathrm{d}$ is the number of the Gaussians ;

- $\sigma_{\mathrm{i}}$ is the deviation standard of the $\mathrm{i}^{\text {th }}$ Gaussian probability density function;

- $\sigma_{\text {Histogram }}$ is the deviation standard of the original histogram.

The acceptance of these two criteria in the threshold algorithm established by the introduction of two parameters: $w_{1}$ and $w_{2}$. So, $w_{1}$ and $w_{2}$ are the weighting parameters and make it possible to reach the limit of the feasible domain. This operation used when the purpose of the segmentation is to isolated the target from the original image.

The objective of this method is to amplify the information on the position of the optimal threshold, which lets us to achieve a good segmentation. In this sequel, we discuss the different criteria that we will maximize later for the multilevel image threshold process; like the Tsallis and the Rényi entropies criterion.

\subsection{Tsallis Entropy Criterion}

Tsallis proposed a new form of entropy built on a generalization to a parameter of the Boltzmann-GibbsShannon entropy [7], which known as "Tsallis entropy". It defined as follows:

$$
S_{\mathrm{q}}=\frac{1-\Sigma_{i=1}^{k}\left(\mathrm{p}_{\mathrm{i}}\right\}^{\mathrm{H}}}{q-1}
$$

Where $\mathrm{k}$ is the total number of possibilities of the system and $\mathrm{q}$ is the real number designating the entropic index, which characterizes the degree of no-extensively.

In the case of an image by K-gray-levels, let $\mathrm{p}_{\mathrm{i}}=\mathrm{p}_{1}, \mathrm{p}_{2} \ldots \mathrm{p}_{\mathrm{K}}$ the probability distribution of the levels. For the gray-levels t, a two-level threshold assumed. In [8], two classes introduced, A and $\mathrm{B}$, and their probability distributions:

$$
\begin{aligned}
& \text { Class A: } \frac{\mathrm{p}_{1}}{\mathrm{p}_{\mathrm{A}}}, \frac{\mathrm{p}_{2}}{\mathrm{p}_{\mathrm{A}}}, \ldots, \frac{\mathrm{p}_{\mathrm{t}}}{\mathrm{p}_{\mathrm{A}}}
\end{aligned}
$$

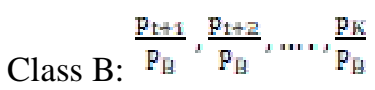

Where

$$
P_{A}=\Sigma_{i=1}^{t} P_{i} \text { and } P_{B}=\Sigma_{i=t+1}^{K} P_{i}
$$

The a priori Tsallis entropy for each distribution defined as:

$$
\begin{aligned}
& S_{q}^{A}=\frac{1-E_{i=1}^{L}\left(\frac{P_{i}}{P_{A}}\right]^{q}}{q-1} \\
& S_{q}^{\mathrm{g}}=\frac{1-Z_{i=L+1}^{K}\left(\frac{P_{i}}{P_{\square}}\right)^{4}}{q-1}
\end{aligned}
$$

Where $S_{\mathrm{q}}(\mathrm{t})$ represent the Tsallis entropy of each individual class who has depended on the threshold t. The Tsallis entropy total of the image written as follows [15]:

$S_{q}(t)=S_{q}^{A}(t)+S_{q}^{\mathrm{g}}(t)+(1-q) \times S_{q}^{A}(t) \times S_{q}^{B}(t)$

In computer vision and image processing, the decrease of a gray level image to a binary image obtained through a clustering-based image thresholding. Let us take a $\mathrm{k}$-grayscale image again, with a distribution of probabilities, $\mathrm{p}_{\mathrm{i}}=\mathrm{p}_{1}, \mathrm{p}_{2} \ldots \mathrm{p}_{\mathrm{K}}$. Let us assume some thresholds $\mathrm{t}_{1}, \mathrm{t}_{2} \ldots \mathrm{t}_{\mathrm{m}}$ for the gray levels. Some classes should be introducing; their probability distributions are by Sparavigna [9] as follow:

(1): $\frac{\frac{p_{1}}{P_{1}}, \frac{p_{Z}}{P_{1}}, \ldots, \frac{P_{t_{1}}}{P_{1}}}{\text { (2): } \frac{P_{t_{1}+1}}{P_{z}}, \frac{P_{t_{1}}=2}{P_{2}}, \ldots, \frac{P_{t_{2}}}{P_{z}}}$

$\cdots$

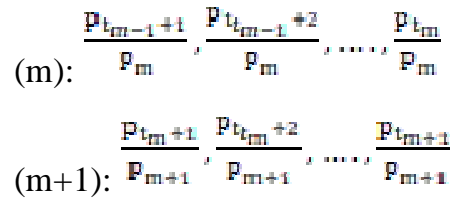

Therefore, we have: 
$\mathrm{S}_{\mathrm{q}}=\mathrm{S}_{\mathrm{q}}^{(1)}+\mathrm{S}_{\mathrm{q}}^{(2)}+\cdots+\mathrm{S}_{\mathrm{q}}^{(\mathrm{m}+1)}+(1-$

q) $=\sum_{i=1}^{m+1} \sum_{j=1 ; i=1}^{m+1} s_{q}^{(i)} s_{q}^{(i)}+(1-$

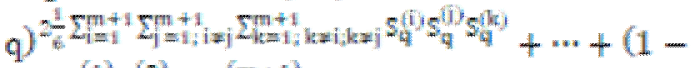
q) ${ }^{m} S_{q}^{(1)} S_{q}^{(2)} \ldots S_{q}^{(m+1)}$

\subsection{Rényi Entropy Criterion}

We take into account the limitations of Shannon measure in certain situations; Rényi [10] took the initial step and generalized the Shannon measure. To tackle the weakness of the Shannon measure in some situations, Rényi entropy took the primary step and proposed a parametric measurement of the information.

In the literature, applications of Rényi's entropy cover several arenas ranging from biology to medicine, through genetics, linguistics and economics, to electrical engineering, computer science, geophysics, chemistry and physics [11].The Rényi's entropy measure of the $\alpha$ order of an image defined as $[11,12]$ :

$\mathrm{H}_{\mathrm{a}}(\mathrm{P})=\frac{1}{1-\mathrm{a}} \ln \sum_{\mathrm{i}=1}^{\mathrm{k}}\left(\mathrm{p}_{\mathrm{i}}\right)^{\mathrm{a}}$

Where $\alpha$ is a positive real parameter and $\alpha \neq 1$. Since Shannon's entropy, measurement is a singular case of the Rényi entropy for $\alpha \rightarrow 1$. Let $\mathrm{p}_{\mathrm{i}}=\mathrm{p}_{1}, \mathrm{p}_{2} \ldots \mathrm{p}_{\mathrm{K}}$ be the probability distribution for a k-grayscale image.

From this distribution, we develop two probability distributions, the first one for the object (class A) and the second one for the background (class B), given by:

Class A: $\frac{\mathrm{P}_{1}}{\mathrm{P}_{\mathrm{A}}}, \frac{\mathrm{P}_{2}}{\mathrm{P}_{\mathrm{A}}}, \ldots, \frac{\mathrm{P}_{\mathrm{t}}}{\mathrm{P}_{\mathrm{A}}}$

Class B: $\frac{P_{t+1}}{P_{B}}, \frac{P_{t+2}}{P_{B}}, \ldots, \frac{P_{k}}{P_{B}}$

Where

$P_{A}=\sum_{i=1}^{t} p_{i}$ and $P_{B}=\sum_{i=t+1}^{k} P_{i}=1-P_{A}$

The Rényi's entropy of order $\alpha$ for each distribution defined as:

$\mathrm{H}_{\mathrm{a}}^{A}(\mathrm{t})=\frac{1}{1-\mathrm{a}} \ln \Sigma_{\mathrm{i}=1}^{\mathrm{t}}\left(\frac{\mathrm{p}_{\mathrm{i}}}{\mathrm{p}_{\mathrm{N}}}\right)^{\mathrm{a}}$

$\mathrm{H}_{\mathrm{a}}^{\mathrm{B}}(\mathrm{O})=\frac{1}{1-\mathrm{a}} \ln \sum_{\mathrm{i}=\mathrm{t}+1}^{\mathrm{k}}\left(\frac{\mathrm{p}_{\mathrm{i}}}{\mathrm{P}_{\mathrm{B}}}\right)^{\mathrm{a}}$

$\mathrm{H}_{\mathrm{a}}(\mathrm{t})$ depends parametrically on the threshold value $\mathrm{t}$ for the object and the background, and written as follows:

$H_{a}(t)=H_{a}^{A}(t)+H_{G}^{B}(t)$

For an image, the whole distribution is divided into $\mathrm{N}$ number of classes $C=\left(C_{1}, C_{2}, C_{3}, \ldots, C_{N}\right)$. Then Rényi's priori for each distribution defined as [10]:

$\mathrm{H}_{\mathrm{a}}\left(\mathrm{C}_{1}\right)=\frac{1}{1-\mathrm{a}} \ln \sum_{\mathrm{i}=1}^{\mathrm{t}_{1}}\left(\frac{\mathrm{p}_{\mathrm{i}}}{\left.\mathrm{p}_{\left(\mathrm{c}_{1}\right)}\right)^{\mathrm{a}}}\right.$

$$
\begin{aligned}
& \mathrm{H}_{\mathrm{a}}\left(\mathrm{C}_{\mathrm{2}}\right)=\frac{1}{1-\mathrm{a}} \ln \sum_{\mathrm{i}=\mathrm{t}_{1}+1}^{\mathrm{t}_{\mathrm{Z}}}\left(\frac{\mathrm{p}_{\mathrm{i}}}{\left.\mathrm{P}_{\left(\mathrm{c}_{2}\right.}\right)^{\mathrm{a}}}\right. \\
& \cdots \\
& \mathrm{H}_{\mathrm{a}}\left(\mathrm{C}_{\mathrm{N}}\right)=\frac{1}{1-\mathrm{a}} \ln \sum_{\mathrm{i}=\mathrm{t}_{\mathrm{N}-1}}^{\mathrm{L}-1}\left(\frac{\mathrm{p}_{\mathrm{i}}}{\mathrm{P}_{\left(c_{\mathrm{N}}\right)}}\right)^{\mathrm{a}}
\end{aligned}
$$

With:

$$
\begin{aligned}
& P\left(C_{1}\right)=\Sigma_{i=1}^{t} p_{i}, P\left(C_{2}\right)=\sum_{i=t+1}^{t_{2}} p_{i}, \ldots, P\left(C_{\mathbb{N}}\right)= \\
& \Sigma i=t_{N-1}^{L-1} p_{i} .
\end{aligned}
$$

Here $\mathrm{p}_{\mathrm{i}}$ is the normalized histogram and $\mathrm{L}$ is the highest grey level intensity.

$$
\mathrm{H}_{\mathfrak{a}}(\mathrm{t})=\mathrm{H}_{\mathbb{a}}\left(\mathrm{C}_{1}\right)+\mathrm{H}_{\mathbb{a}}\left(\mathrm{C}_{2}\right)+\cdots+\mathrm{H}_{\mathbb{a}}\left(\mathrm{C}_{\mathbb{N}}\right)
$$

\section{EVALUATION OF CRITERIAN}

In this work, the implementation of the new multi-objective optimization technique founded on the Rényi and the Tsallis entropies requires a reliable quantitative and qualitative evaluation. For this, we present in this section the evaluation criteria: namely the Levin and Nazif criterion and the mean square error criterion.

\subsection{Levine and Nazif Criterion}

\section{a. Intra-region uniformity}

One of the most intuitive criteria for quantifying the eminence of a segmentation result is the intra-region uniformity. Allowing to Weszka et al. [13], Levine and Nazif [16] and El Joumani et al [5] the criterion of intra-region uniformity is as follows:

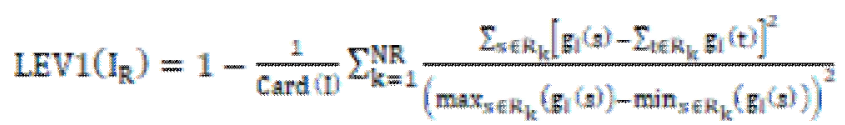

Where

- $\mathrm{I}_{\mathrm{R}}$ corresponds to the segmentation result of the image in a set of regions $\mathrm{R}=\left\{\mathrm{R}_{1}, \ldots, \mathrm{R}_{\mathrm{NR}}\right\}$ having $\mathrm{NR}$ regions,

- $\operatorname{Card}(\mathrm{I})$ corresponds to the number of pixels of the image I,

- $\mathrm{g}_{\mathrm{I}}(\mathrm{s})$ corresponds to the gray-level intensity of the pixels of the image I and can be generalized to any other characteristic (color, texture ...).

\section{b. Inter-region disparity}

Complementary to the intra-region uniformity, Levine and Nazif defined a disparity measurement between two regions to assess the dissimilarity of regions in a segmentation result [16]. The formula for total disparity between regions defined as follows: 


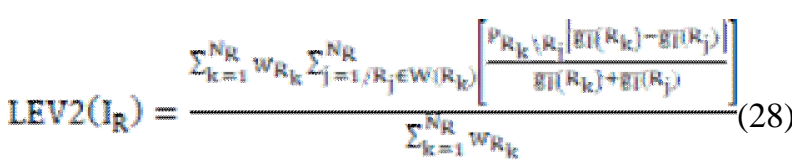

Where $w_{\mathbb{R}_{\mathrm{R}}}$ is a weight associated to $\mathrm{R}_{\mathrm{k}}$ that can be dependent of its area, for example, $\overline{g_{k}}$ is the average of the gray-level of $\mathrm{R}_{\mathrm{k}} \cdot \overline{g_{h}}\left(R_{h}\right)$ can be generalized to a feature vector computed on the pixels values of the region $R_{k}$ such as for LEV1. $P_{R_{h} \backslash R_{j}}$ corresponds to the length of the perimeter of the region $\mathrm{R}_{\mathrm{k}}$ common to the perimeter of the region $\mathrm{Rj}$. This type of criterion has the advantage of penalizing the over segmentation. (Formula intra-inter region)

\section{c. The intra-inter-region dissimilarity}

Note that the intra-region uniformity can combined with the inter-region dissimilarity by using the following formula:

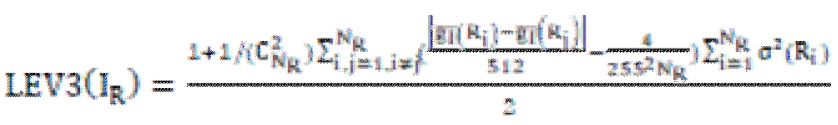

Where $C_{W_{R}}^{2}$ is number of combinations of two regions among $\mathrm{N}_{\mathrm{R}}$.

This criterion combines intra-region and inter-region disparities. Intra-region disparity calculated by the normalized standard deviation of gray levels in each region. The inter-region disparity calculates the dissimilarity of the average gray level of two regions in the segmentation result.

\subsection{Mean Squared Error Criterion}

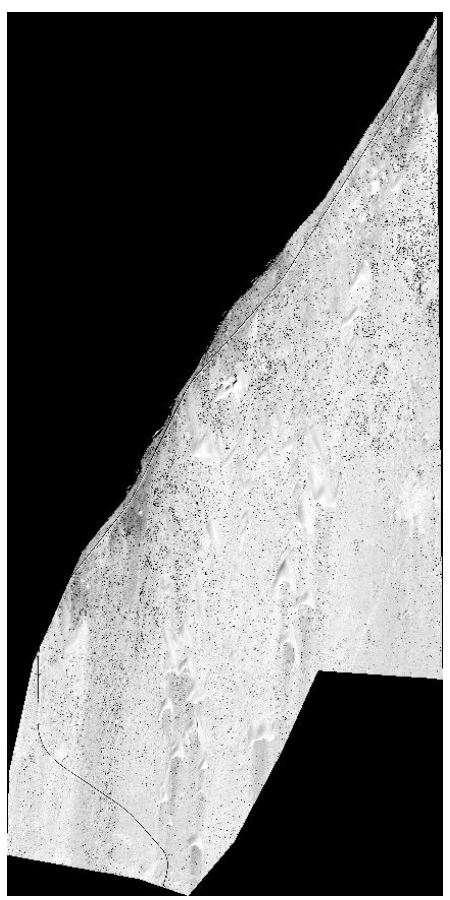

Figure 1: Area of study - Worldview Image
Mean Square Error criterion (MSE) is computed pixel-by-pixel by adding up the squared difference of all the pixels and dividing by the total pixel count. MSE of the segmented image can calculated by using the equation given by Gobindchandra et al. in [14] as below:

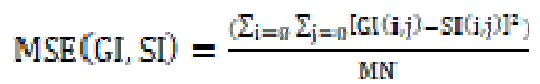

Where, $\mathrm{M}$ and $\mathrm{N}$ are the number of rows and columns in the input image respectively. While, $G I$ and $S I$ are the original and segmented image. MSE must have a lower value to have a higher quality segmented image.

\section{WORLDVIEW IMAGE DATA}

The Worldview image (Fig. 1) retained for this work is a selected zone of Laâyoune-Sakia-el-Hamra province, southwest of Morocco, delimited by longitude $\varphi=-13^{\circ} 35^{\prime} 62.51^{\prime \prime} \mathrm{W}$ and latitude $\lambda=27^{\circ} 37^{\prime} 49.30^{\prime \prime} \mathrm{N}$. This image captured on February 16, 2013 at 11:45:25 pm. We used the panchromatic band (see the characteristic in Table 1). For this work, we are interested in a variety of samples of the Worldview image and red counters on the image (Fig. 2) delimit these selected areas of interest.

Table 1: Characteristic of Panchromatic Worldview image

\begin{tabular}{lll}
\hline Band & $\begin{array}{l}\text { Wavelength } \\
(\mathbf{n m})\end{array}$ & $\begin{array}{l}\text { Spatial } \\
\text { resolution }\left(\mathbf{m}^{2}\right)\end{array}$ \\
\hline Panchromatic & $450-800$ & $0.55 \times 0.55$ \\
\hline
\end{tabular}

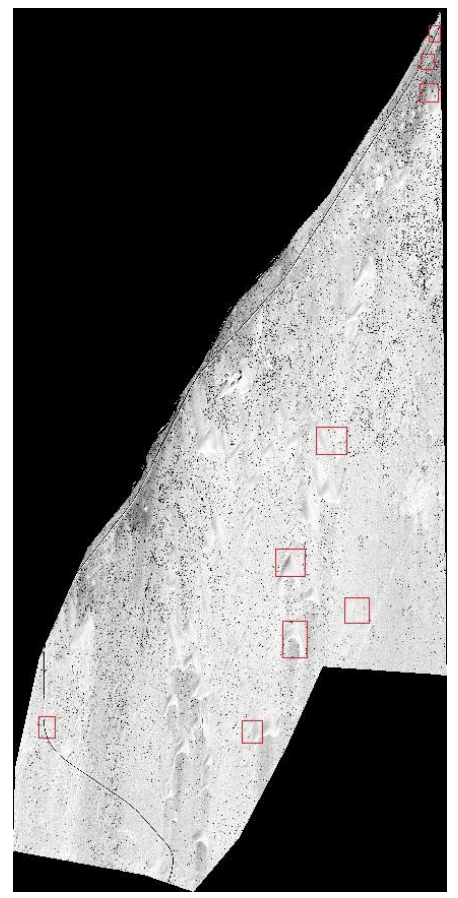

Figure 2: Worldview Image - Zone of Interest 


\section{EXPERIMENTAL RESULTS AND DISCUSSION}

To improve our algorithms, we test at first the synthetic images of gray level types, the synthetic images taken from database [17] and we validated at second for the very high spatial resolution satellite images of Worldview. For this work, $\mathrm{q}=\alpha=0.5$ is considered in both formulas (Eq. 14) and (Eq. 26).

In the first phase of experimentation, we tried on the synthetic images to evaluate the method provided. To study the influence of small regions, the first image we preferred contained a texture. We observed that the values of the inter-region, intra-inter-region and MSE criteria of our proposed method are lower than those provided by the method [5], and the intra-region criterion values are superior. The same findings obtained when processing other synthetic images having different morphological properties. Figure 3 presents the segmented results for synthetic images. In addition, the table 2 illustrate the results obtained by our method and their challenging with the method [5]; it authenticates that the good results obtained by our developed method.

In the second experimental phase, we applied the algorithm to a Worldview image. By adjusting the threshold and the filtering coefficients to segment each image, we also compute their centers of gravity as well as their evaluation for Levine and Nazif criterion and the mean squared error criterion MSE for the proposed method and the comparative method. To evaluate the quality of the segmentation results of the real images, which habitually covers numerous unidentified degradations, the second phase of this comparative experimental study conducted accordingly and evaluated using a real gray level image and of a set of VHSR satellite images. And from the segmentation images (see Figure 4) and the evaluation criterion values obtained in the table (Table 3 ), which were consistently lower than those obtained when using the method [5], the multi-objective optimization provides more stable and reliable results, especially in the case of high-resolution satellite images.

Table 2: Values of Levine and Nazif and MSE evaluation of criteria for synthetic images

\begin{tabular}{|c|c|c|c|c|c|c|c|c|c|c|}
\hline \multirow{2}{*}{ Imagery } & \multirow{2}{*}{ Threshold } & \multirow{2}{*}{ filter } & \multicolumn{2}{|c|}{$\begin{array}{l}\text { Intra-region criterion } \\
\text { of Levine and Nazif }\end{array}$} & \multicolumn{2}{|c|}{$\begin{array}{l}\text { Inter-region criterion } \\
\text { of Levine and Nazif }\end{array}$} & \multicolumn{2}{|c|}{$\begin{array}{l}\text { Intra-inter criterion } \\
\text { region of Levine and } \\
\text { Nazif }\end{array}$} & \multicolumn{2}{|c|}{ MSE } \\
\hline & & & $\begin{array}{l}\text { Previous } \\
\text { method }\end{array}$ & $\begin{array}{l}\text { Proposed } \\
\text { method }\end{array}$ & $\begin{array}{l}\text { Previous } \\
\text { method }\end{array}$ & $\begin{array}{l}\text { Proposed } \\
\text { method }\end{array}$ & $\begin{array}{l}\text { Previous } \\
\text { method }\end{array}$ & $\begin{array}{l}\text { Proposed } \\
\text { method }\end{array}$ & $\begin{array}{l}\text { Previous } \\
\text { method }\end{array}$ & $\begin{array}{l}\text { Proposed } \\
\text { method }\end{array}$ \\
\hline $\begin{array}{l}\text { ImagSynt } \\
1\end{array}$ & 3 & 3 & 0.0097 & 0.0110 & 0.1555 & 0.1497 & 0.5318 & 0.5295 & $1.0714 \mathrm{e}+04$ & $1.0702 \mathrm{e}+04$ \\
\hline $\begin{array}{l}\text { ImagSynt } \\
2\end{array}$ & 3 & 3 & 0.0023 & 0.0024 & 0.1865 & 0.1835 & 0.5204 & 0.5195 & $2.6643 \mathrm{e}+03$ & $2.6575 \mathrm{e}+03$ \\
\hline $\begin{array}{l}\text { ImagSynt } \\
3\end{array}$ & 0.01 & 0 & 0.0043 & 0.0124 & 0.3301 & 0.2495 & 0.5394 & 0.5387 & $5.5009 e+03$ & $5.5000 \mathrm{e}+03$ \\
\hline $\begin{array}{l}\text { ImagSynt } \\
4\end{array}$ & 4 & 1 & 0.0590 & 0.0760 & 0.2880 & 0.2485 & 0.5398 & 0.5687 & $1.6601 \mathrm{e}+04$ & $1.6568 \mathrm{e}+04$ \\
\hline $\begin{array}{l}\text { ImagSynt } \\
5\end{array}$ & 3 & 0 & 0.0117 & 0.0299 & 0.2187 & 0.1712 & 0.5408 & 0.5397 & $6.4119 e+03$ & $6.3860 \mathrm{e}+03$ \\
\hline $\begin{array}{l}\text { ImagSynt } \\
6\end{array}$ & 2 & 1.5 & 0.0143 & 0.0421 & 0.2057 & 0.1811 & 0.5493 & 0.5483 & $9.4947 \mathrm{e}+03$ & $9.4334 e+03$ \\
\hline
\end{tabular}

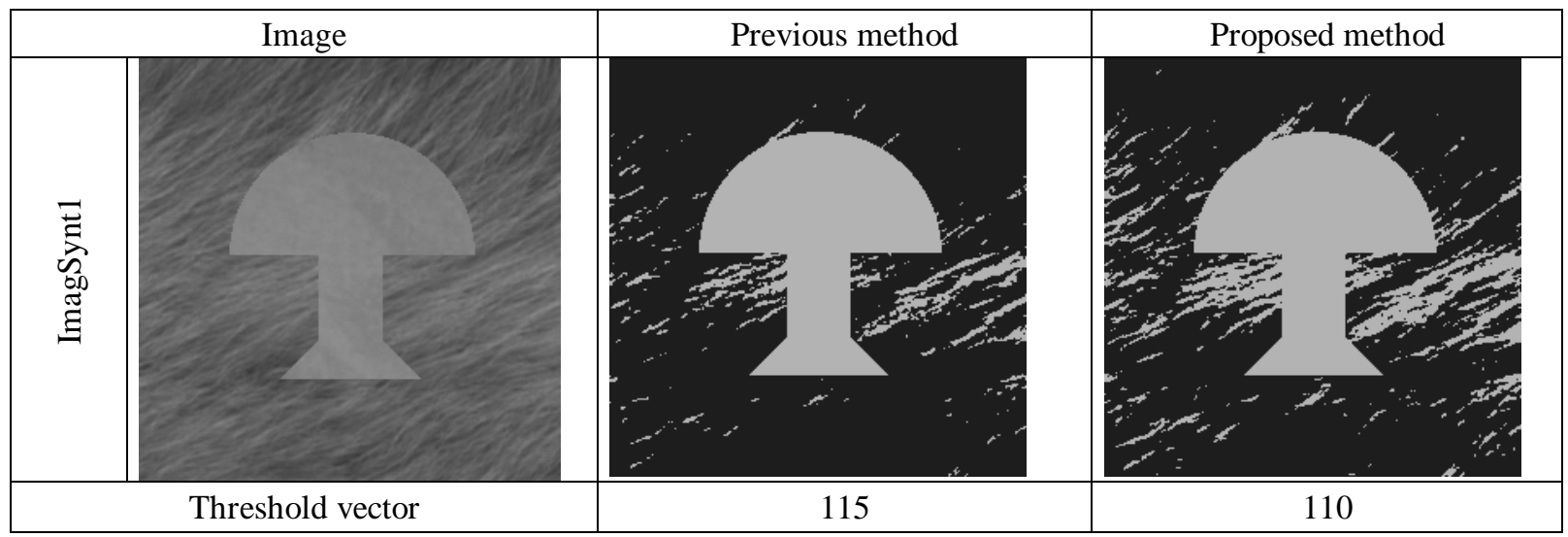




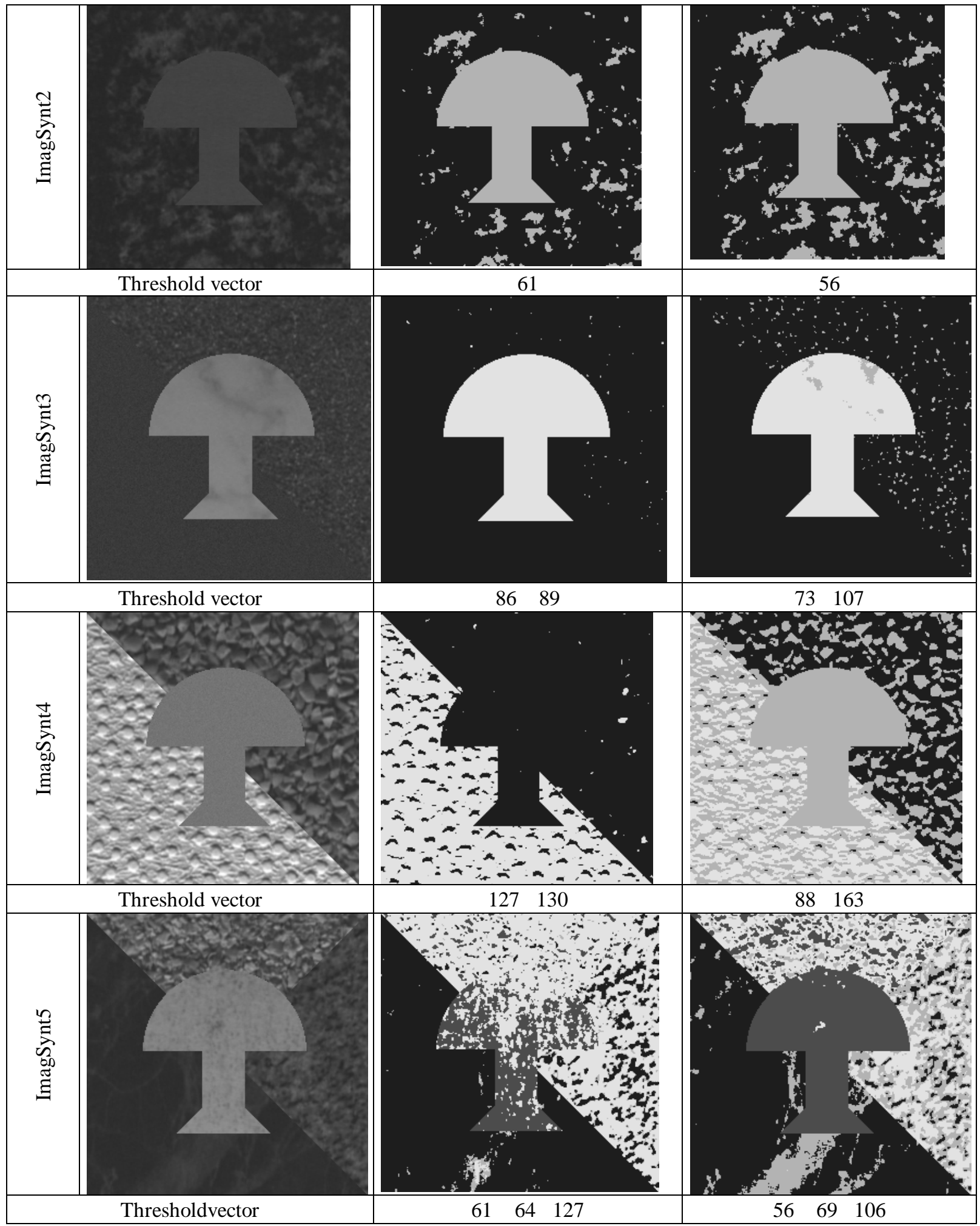




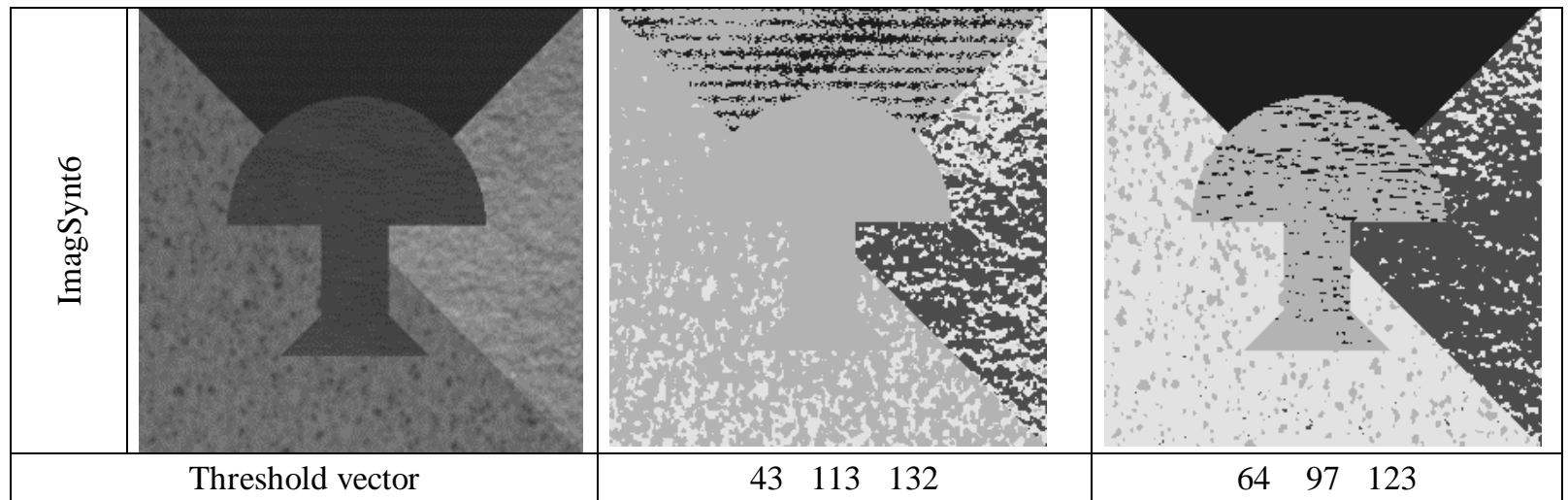

Figure 3: Segmentation result for synthetic images;

Table 3 :Values of Levine and Nazif and MSE evaluation of criteria for panchromatic images

\begin{tabular}{|c|c|c|c|c|c|c|c|c|c|c|}
\hline \multirow{2}{*}{ Imagery } & \multirow{2}{*}{ Threshold } & \multirow{2}{*}{ filter } & \multicolumn{2}{|c|}{$\begin{array}{c}\text { Intra-region } \\
\text { criterion of Levine } \\
\text { and Nazif }\end{array}$} & \multicolumn{2}{|c|}{$\begin{array}{l}\text { Inter-region criterion } \\
\text { of Levine and Nazif }\end{array}$} & \multicolumn{2}{|c|}{$\begin{array}{c}\text { Intra-inter } \\
\text { criterion region of } \\
\text { Levine and Nazif }\end{array}$} & \multicolumn{2}{|c|}{ MSE } \\
\hline & & & $\begin{array}{l}\text { Previous } \\
\text { method }\end{array}$ & $\begin{array}{l}\text { Proposed } \\
\text { method }\end{array}$ & $\begin{array}{l}\text { Previous } \\
\text { method }\end{array}$ & $\begin{array}{l}\text { Proposed } \\
\text { method }\end{array}$ & $\begin{array}{l}\text { Previous } \\
\text { method }\end{array}$ & $\begin{array}{l}\text { Proposed } \\
\text { method }\end{array}$ & $\begin{array}{l}\text { Previous } \\
\text { method }\end{array}$ & $\begin{array}{l}\text { Proposed } \\
\text { method }\end{array}$ \\
\hline ImagSat1 & 4 & 1.5 & 0.1185 & 0.1309 & 0.1284 & 0.1131 & 0.5354 & 0.5317 & $4.2118 \mathrm{e}+04$ & $4.1987 e+04$ \\
\hline ImagSat2 & 3.9 & 1.5 & 0.1319 & 0.1398 & 0.1619 & 0.1476 & 0.5480 & 0.5411 & $3.5385 \mathrm{e}+04$ & $3.5299 \mathrm{e}+04$ \\
\hline ImagSat3 & 5 & 1.5 & 0.2283 & 0.2288 & 0.1741 & 0.1264 & 0.5315 & 0.5230 & $4.3872 \mathrm{e}+04$ & $4.3730 \mathrm{e}+04$ \\
\hline ImagSat4 & 4.6 & 1.5 & 0.0967 & 0.0965 & 0.1268 & 0.1032 & 0.5442 & 0.5385 & $4.4796 e+04$ & $4.4679 \mathrm{e}+04$ \\
\hline ImagSat5 & 3 & 0 & 0.2019 & 0.2345 & 0.0938 & 0.0920 & 0.5207 & 0.5195 & $4.7283 e+04$ & $4.7149 \mathrm{e}+04$ \\
\hline ImagSat6 & 2 & 1.5 & 0.0641 & 0.0693 & 0.0730 & 0.0719 & 0.5340 & 0.5319 & $4.3321 \mathrm{e}+04$ & $4.3261 \mathrm{e}+04$ \\
\hline
\end{tabular}

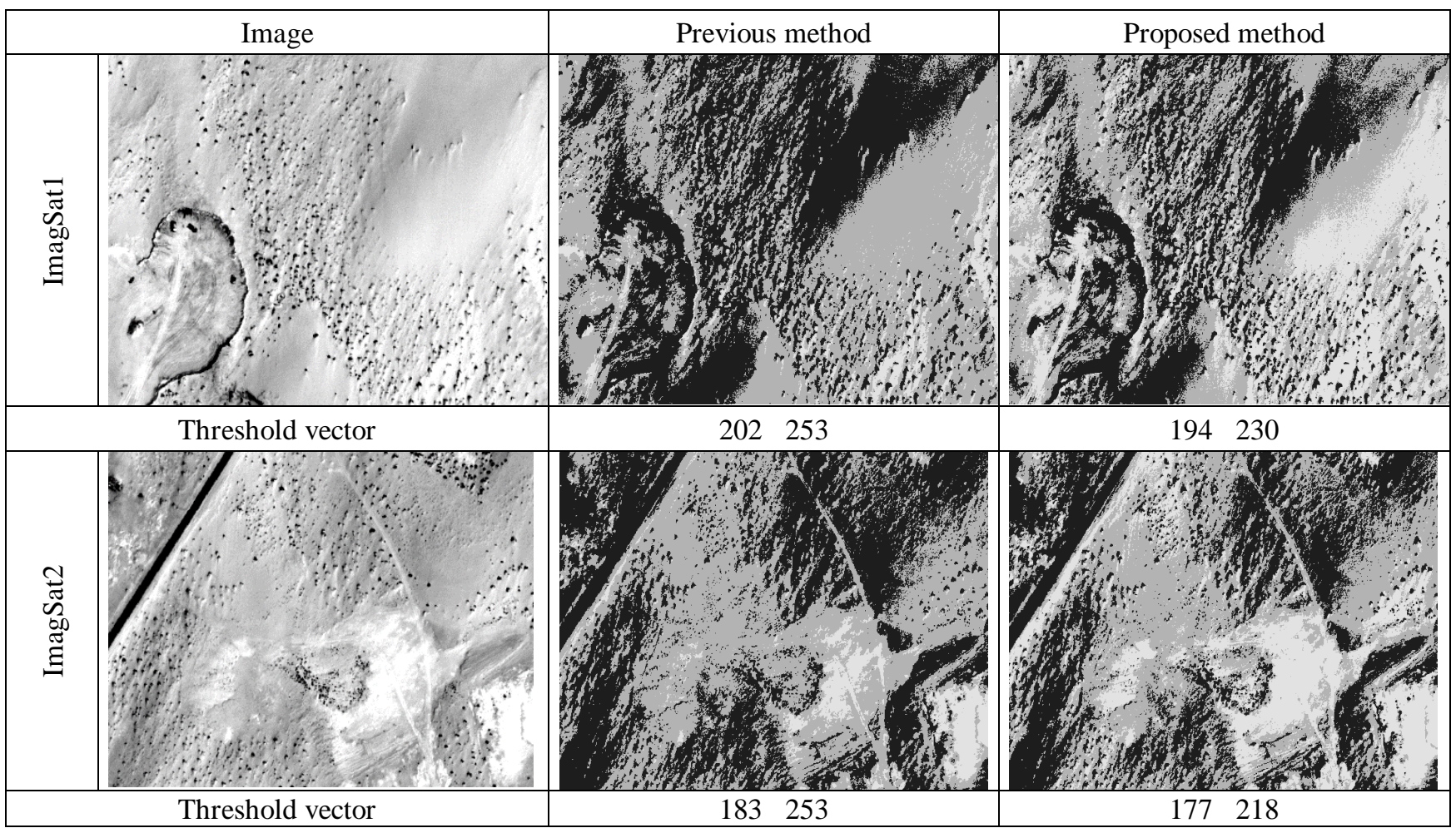




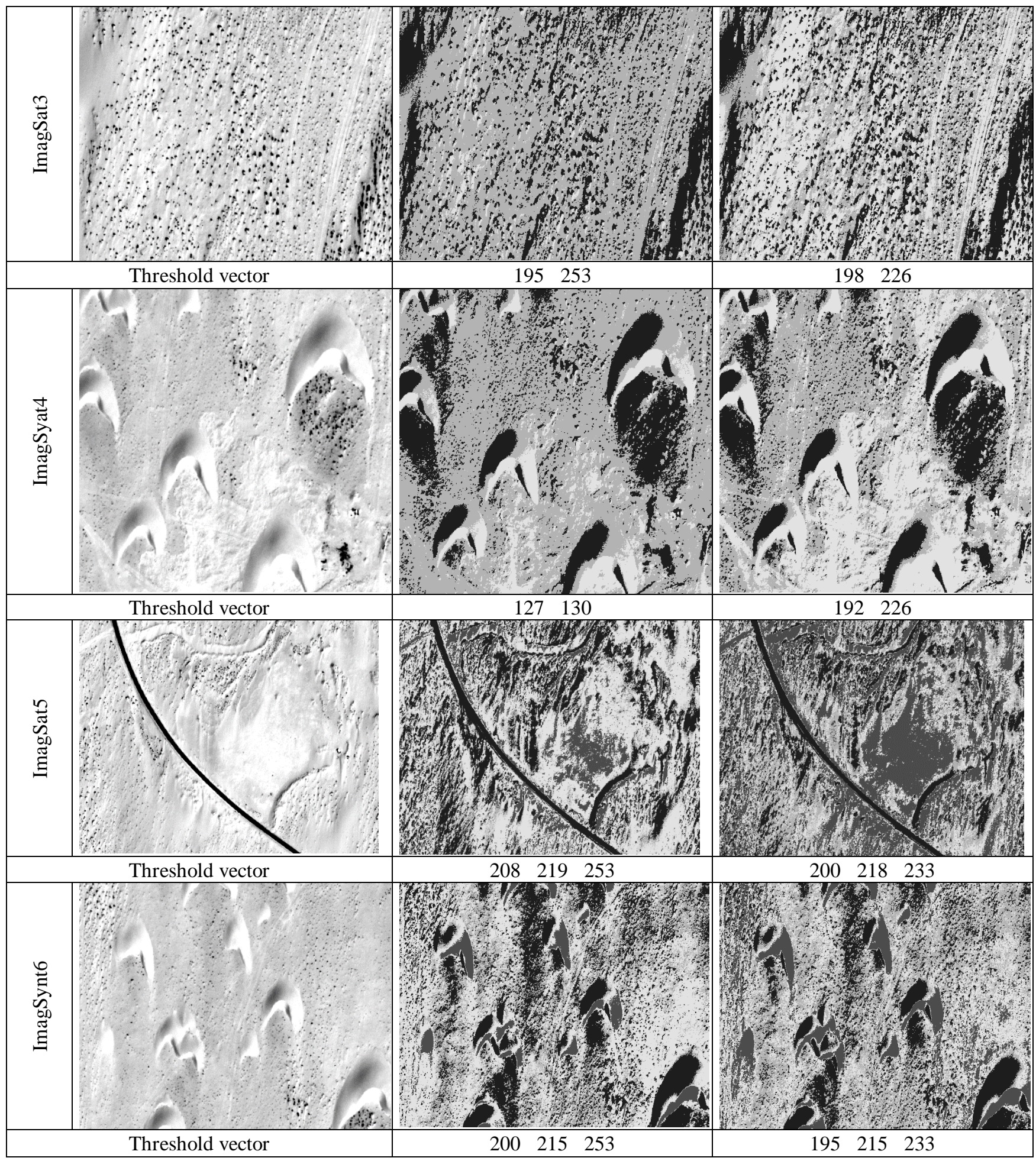

Figure 4: The Segmentation result for panchromatic images;

According to the evaluation, criteria of Levin and Nazif and the mean squared error and following the comparison of the results obtained by the two methods. We clearly distinguish both for the synthesis images and for the satellite images that our proposed method: Multi-Objective Optimization for Worldview Image Segmentation Funded on the Entropies of Tsallis and Rényi, is much better than that of El Joumani et al [5]. This shows the novelty of our proposed method and confirms that our research work is obviously a significant progress.

\section{THE INFLUENCE OF PARAMETERS Q AND A IN IMAGE SEGMENTATION}

Image thresholding based on Rényi's entropy and image thresholding based on Tsallis entropy are two important 
approaches to selecting the global threshold for image segmentation. In fact, they can provide equivalent results, that is, the two approaches give the same threshold when Sparavigna chose the same parameter in [18].

According to Table 4 at a result of changing of the coefficient $q=\alpha$, the result of the specified criterion changed the norm of the segmentation quality as well for both the synthetic image "ImagSyn5" and the satellite image "ImageSat5", and thus, according to Figure 5 we find the same result for both images ("ImagSyn5" and "ImagSat5").
We ascertain that $(\mathrm{q}=\alpha)$ was the sole specified parameter identifying the segmentation quality.

Table 4: Different result of the specified criterion with $\mathrm{q}=\alpha$

\begin{tabular}{lllllllll}
\hline \multirow{2}{*}{$\mathrm{q}=\alpha$} & \multicolumn{1}{l}{ ImgSynt5 } & \multicolumn{7}{c}{ ImagSat5 } \\
\cline { 2 - 10 } & LEV1 & LEV2 & LEV3 & MSE & LEV1 & LEV2 & LEV3 & MSE \\
\hline 0.25 & 0.1716 & 0.0124 & 0.5402 & $6.3825 \mathrm{e}+03$ & 0.0920 & 0.2345 & 0.5195 & $4.7149 \mathrm{e}+04$ \\
\hline 0.50 & 0.1704 & 0.0120 & 0.5400 & $6.3791 \mathrm{e}+03$ & 0.0920 & 0.2345 & 0.5195 & $4.7149 \mathrm{e}+04$ \\
\hline 0.75 & 0.1714 & 0.0118 & 0.5397 & $6.3804 \mathrm{e}+03$ & 0.0982 & 0.2351 & 0.5244 & $4.7326 \mathrm{e}+04$ \\
\hline 1.25 & 0.2380 & 0.0385 & 0.5218 & $6.3525 \mathrm{e}+03$ & 0.0910 & 0.1755 & 0.5128 & $4.7200 \mathrm{e}+04$ \\
\hline
\end{tabular}

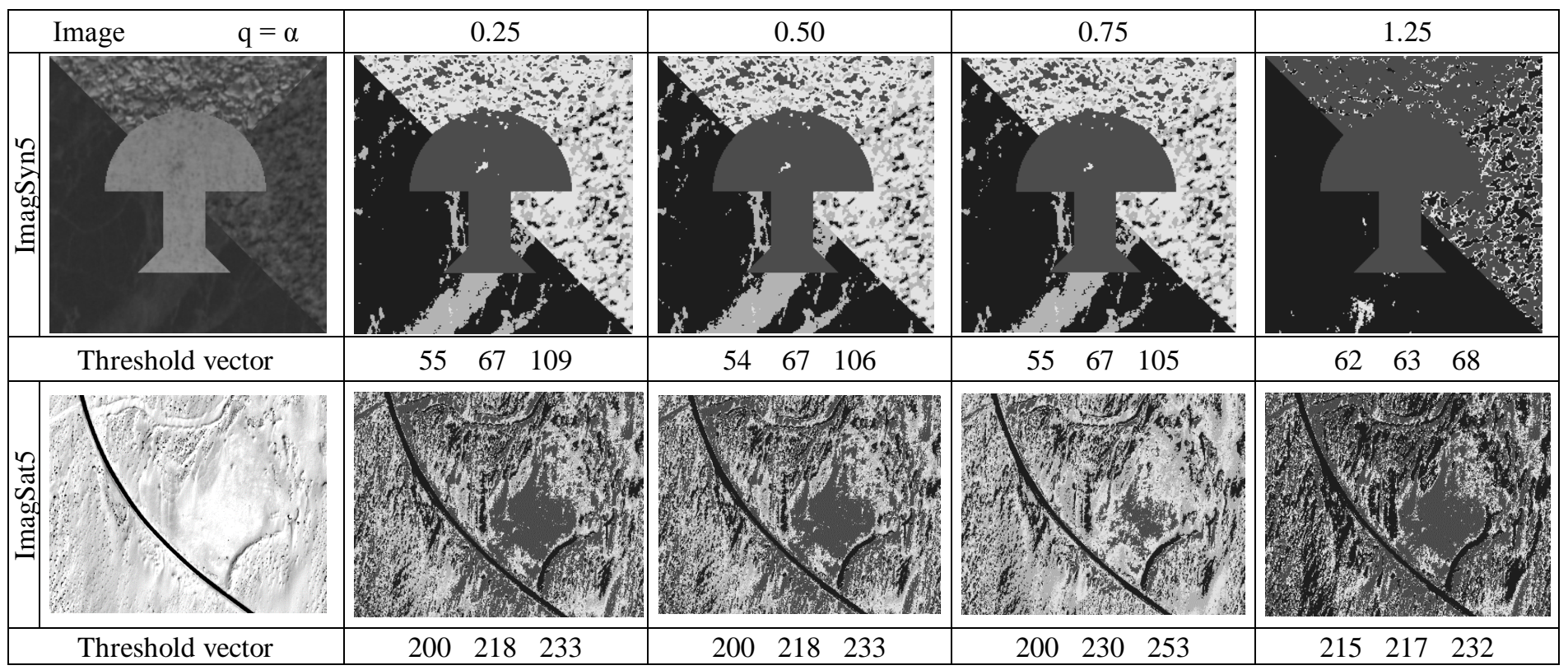

Figure 5: Different segmentation result of the specified criterion with $q=\alpha$;

\section{CONCLUSION}

In this work, we have proposed a new multi-objective optimization method for image segmentation based on the entropies of Tsallis and Rényi. We applied this method on VHSR satellite images, which allows optimal separation of the different classes of gray levels in an optimal way according to some criteria.

We applied our method according to the segmentation of multiclass images such as synthetic images and the
Worldview image samples in order to evaluate the proposed function with respect to the previous method [5]. The evaluation of the segmentation by the introduction of Levine and Nazif evaluation criteria and the mean square error shows that the new method developed gives the better result than the previous method [5].

\section{ACKNOWLEDGEMENT}

A COMSTECH TWAS 2015 Research Grant Award supported the satellite image used for this work partially. 


\section{REFERENCES}

1. S. E Mechkouri., R. Zennouhi, S. El Joumani, L. Masmoudi, J. Gonzalez; Quantum segmentation approach for very high spatial resolution satellite image: application to QuickBird image. Journal of Theoretical and Applied Information Technology. Vol. 62(2), pp. 539-545. April 2014

2. S. E Mechkouri., R. Zennouhi, L. Masmoudi, J. Gonzalez. Colour image segmentation using hierarchical analysis of 2D-histograms: application to urban land cover and land use classification. Geo-Observateur 18, pp. 43-57. March 2010

3. R. Zennouhi, L. Masmoudi. A new 2D-histogram scheme for colour image segmentation. Imaging Sci. J. Vol. 57, pp. 260-365, 2009.

https://doi.org/10.1179/136821909X12490307952874

4. D. Sarala, T. Kanikdaley, S. Jogi , R. K. Chaurasiya. Content-Based Image Retrieval Using Hierarchical Color and Texture Similarity Calculation. International Journal of Advanced Trends in Computer Science and Engineering.

Vol. 7 No 2, pp. 11-16, 2018

https://doi.org/10.30534/ijatcse/2018/02722018

5. S. El Joumani, S.E. Mechkouri, R. Zennouhi, O. El Kadmiri, L. Masmoudi. Segmentation method based on multiobjective optimization for very high spatial resolution satellite images. EURASIP Journal on Image and Video Processing, DOI 10.1186/s13640-016-0161-2. 2017

6. A. Nakib, H. Oulhadj, P. Siarry. Image histogram thresholding based on multiobjective optimization. Signal Process, 87, pp. 2516-2534, 2007

https://doi.org/10.1016/j.sigpro.2007.04.001

7. C. Tsallis. Possible generalization of Boltzmann-Gibbs statistics. Journal of Statistical Physics, 52, pp. 479-487, 1988

8. M. Portes de Albuquerque, I.A. Esquef, Gesualdi A.R. Mello,. Image thresholding using Tsallis entropy. Pattern Recognition Letter, 25(9), pp. 1059-1065, 2004.

9. A.C. Sparavigna, Tsallis entropy in bi-level and multi-level image thresholding, International Journal of Sciences. 4(1), pp. 40-49, 2015.

https://doi.org/10.18483/ijSci.613

10. A Rényi, On measures of entropy and information.

In: Neyman, J., Ed., 4th Berkeley Symposium on Mathematical Statistics and Probability, Berkeley, 1, 547-561, 1961.

11. M. A. El-Sayed, M. A. Khafagy, Using Rényi's entropy for edge detection in level images. International Journal of Intelligent Computing and Information Science, Vol. 11, No. 2, JULY 2011.

12. P. Sahoo, C. Wilkins and J. Yeager. Threshold Selection Using Rényi's Entropy. Pattern Recognition, pp. 71-84, 1997.

13. JS. Weszka, A. Rosenfeld. Threshold evaluation techniques. IEEE Trans. Syst. Man. Cybern. Vol. 8, No 8, pp. 622-629, 1978.
https://doi.org/10.1109/TSMC.1978.4310038

14. K. Gobindchandra, K.L. Santhosh Kumar. Analysis of Image Segmentation Techniques. International Research Journal of Computer Science, Vol. 2 No 6, pp 45-53, 2015. 15. C. Rosenberger, Mise en œuvre d'un système adaptatif de segmentation d'images, $\mathrm{PhD}$. thèses Université de Rennes1, Rennes. 1999.

16. M. D. Levine, A.M. Nazif. Dynamic measurement of computer generated image segmentations. IEEE Trans. Pattern Anal. Mach. Intell. Vol. 7 No 2, pp. 155-164, 1985.

17. The sample images are taken from the web-site and Available

from:

http://pages.upf.pf/Sebastien.Chabrier/ressources.php http://pages.upf.pf/Sebastien.Chabrier/download/ImSynth.zi $\mathrm{p}$

18. A.C. Sparavigna, On the Role of Tsallis Entropy in Image Processing. International Scientific Research Journal, IRJ. Science, Vol. 1 No 6, pp. 16-24, 2015. https://doi.org/10.18483/IRJSci.79 